\title{
Sistematização da assistência de enfermagem: o conhecimento da equipe de enfermagem de um centro cirúrgico
}

Resumo: Pesquisa qualitativa descritiva que objetivou identificar o conhecimento da equipe de Enfermagem de um Centro Cirúrgico sobre a Sistematização da Assistência de Enfermagem. A coleta dos dados ocorreu por meio de uma entrevista semiestruturada, com enfermeiros, técnicos e auxiliares de Enfermagem, seguida da análise de conteúdo. Emergiram duas categorias: (Des)Conhecimento da equipe de Enfermagem sobre a Sistematização da Assistência de Enfermagem; e o dia a dia da equipe de Enfermagem e sua influência na aplicação da Sistematização da Assistência de Enfermagem. Percebeu-se de entendimento dos participantes sobre a SAE, sendo esta uma das principais ferramentas de trabalho da equipe de Enfermagem.

Descritores: Processos de Enferm., Enfermagem perioperatória, Equipe de enfermagem

\section{Systematization of nursing care: the knowledge of the nursing team at a surgical center}

Abstract: This is a qualitative study that aims to identify the knowledge of the nursing team at a surgical center concerning the systematization of nursing care the data were collected through semi-structured interviews with nurses, technicians and auxiliary nursing staff, followed by an analysis. Two thematic categories emerged: understanding/lack of knowledge of the team concerning systematization of nursing care and the daily life of the nursing team and its influence on the application of systematization of nursing care. There was a lack of knowledge regarding systematization of nursing care on the part of the interviewees, and this raises concerns given that this is an important tool in the work of the nursing team.

Descriptors: Nursing processes, Perioperative nursing, Nursing team

\section{Sistematización de la asistencia de enfermería: el conocimiento del equipo de enfermería de un centro quirúrgico}

Resumen: Estudio cualitativo, con el objetivo de identificar el conocimiento del equipo de enfermería de un Centro Quirúrgico sobre la sistematización de la asistencia de enfermería. La recolección de los datos ocurrió por medio una entrevista semiestructurada, con enfermeros, técnicos y auxiliares de enfermería, seguida del análisis de las mismas según. Emergieron dos categorías temáticas: Entendimiento/desconocimiento del equipo de enfermería sobre la sistematización de la asistencia de enfermería; el día a día del equipo de enfermería y su influencia en la aplicación de la sistematización de la asistencia de enfermería. Se notó un desconocimiento de los entrevistados sobre la SAE, un hecho preocupante, visto que esta es una importante herramienta de trabajo del equipo de enfermería.

Descriptores: Procesos de Enfermería, Enfermería perioperatoria, Grupo de enfermería

\section{INTRODUÇÃO}

$\mathrm{O}$ processo de Enfermagem é um método utilizado para implantar na prática profissional uma teoria de Enfermagem. Após a escolha da teoria de Enfermagem, torna-se necessária a utilização de um método científico para que os conceitos da teoria sejam aplicados ${ }^{(1)}$. A teoria deve ser fundamentada e escolhida de acordo com as condições do cliente, da instituição ou do profissional que irá utilizá-la(2).

No Brasil, Wanda de Aguiar Horta foi à primeira teórica a apresentar estudos sobre o tema. Ela propôs a sistematização das ações de Enfermagem, por meio da aplicação do processo de Enfermagem, o que contribuiu para o registro e documentação de ocorrências e procedimentos realizados por integrantes da equipe de Enfermagem, para a análise e organização do cuidado prestado e, para o reconhecimento social da profissão $o^{(3-4)}$.

O Conselho Federal de Enfermagem (COFEN) considera que a implantação da Sistematização da Assistência de Enfermagem (SAE) contribui, efetivamente, para a melhoria da qualidade e deve ser aplicada em todos os clientes, com prioridade àqueles críticos. Deve ser utilizada por toda instituição de saúde, como atividade privativa do enfermeiro(5), e em todos os setores hospitalares.

O Centro Cirúrgico (CC) é normalmente conhecido como uma unidade estressante, que demanda do enfermeiro maior tempo com serviços administrativos do setor, que às vezes, o faz esquecer-se da assistência direta ao cliente em um momento único de sua vida, em que a instabilidade emocional domina-o.

Por conseguinte, o desenho desta pesquisa é baseado nas etapas da SAE recomendadas pelo COFEN ${ }^{(5)}$, que são: coleta de dados, diagnósticos, planejamento, implementação e avaliação, ressaltando que planejamento e implementação fazem parte da prescrição de Enfermagem.

Visto a importância de se realizar a SAE de forma a contribuir para uma assistência integral e individualizada ao cliente cirúrgico, estabeleceu-se a seguinte questão norteadora: Qual o conhecimento da equipe de Enfermagem de um CC sobre a SAE? No intuito de responder a essa questão o objetivo do trabalho é descrever o conhecimento da equipe de Enfermagem de um CC sobre a SAE.

\section{METODOLOGIA}

Trata-se de uma pesquisa descritiva de abordagem qualitativa. As pesquisas de abordagem qualitativa fundamentam-se na premissa de que o conhecimento sobre as pessoas só é possível a partir da descrição da experiência humana tal como ela é vivida e tal como é definida pelos seus próprios atores ${ }^{(6)}$. 
A pesquisa foi realizada em um hospital de ensino de médio porte, localizado na cidade de Curitiba - PR, no setor de CC. Esta unidade conta com sete salas operatórias com média de 30 cirurgias diárias.

Os participantes do estudo foram técnicos e auxiliares de Enfermagem, e enfermeiros, dos turnos da manhã, tarde e noite. Como critérios de inclusão elencaram-se: equipe de Enfermagem lotados no CC; que ali trabalhassem por período igual ou superior a seis meses, e que aceitassem participar da pesquisa. Foram excluídos àqueles que não atenderam as exigências descritas.

A coleta de dados aconteceu nos meses de agosto e setembro de 2012, por meio de entrevistas semiestruturadas que versaram ou sobre o conhecimento da equipe acerca da $\mathrm{SAE}$, a aplicação desta no CC, sua a influência nos cuidados ao cliente, e as dificuldades encontradas em aplicá-la.

Primeiramente realizou-se um teste piloto em outro setor do hospital, com cinco participantes, visando à avaliação e possível adaptação do instrumento. Por meio desta análise retirou-se uma questão que impossibilitava o desdobramento posterior da entrevista.

Para dar início a coleta de dados, foi acordado com o enfermeiro do CC os horários e datas que propiciassem maior participação dos funcionários. As entrevistas foram realizadas no período da manhã, tarde e noite, com 14 funcionários de um total de 35, conforme disponibilidade e aceitação em participar do estudo. $O$ registro das entrevistas foi realizado por meio de gravação digital e posterior transcrição.

Para a análise dos dados foi utilizada a metodologia de Análise de Conteúdo ${ }^{(7)}$, a qual pela técnica da análise categorial fez-se o desmembramento do texto em unidades e em categorias.

Para a identificação dos profissionais foram utilizados letras e números, sendo TE para os técnicos de Enfermagem, AE para os auxiliares de Enfermagem e a letra $E$ para os enfermeiros. A cada sigla foi acrescido um número para individualização das falas.

A pesquisa foi aprovada pelo Comitê de Ética em Pesquisa da Universidade Positivo com o parecer 77803/2012. Foi também solicitada e concedida autorização da instituição sede do estudo. Nesta investigação foi solicitada a participação da equipe de Enfermagem de forma voluntária. Todas as informações e esclarecimentos a respeito desta, foram prestados aos participantes e após a assinatura do Termo de Consentimento Livre e Esclarecido, deu-se início a coleta dos dados.

\section{RESULTADOS}

Os participantes do estudo foram 14 profissionais, sendo três enfermeiros, nove técnicos e dois auxiliares de Enfermagem. Destes, dois eram do sexo masculino e doze do sexo feminino. A faixa etária foi de 22 a 55 anos, com média de 34,85 anos.

Por meio das entrevistas emergiram duas categorias relacionadas ao conhecimento da equipe de Enfermagem.

\section{(Des) Conhecimento da equipe de Enfermagem sobre a SAE}

Nas entrevistas os profissionais de nível médio apresentaram dificuldade em definir corretamente a SAE, fato evidenciado por respostas incertas ou confusas, pois ao serem questionados sobre seu conhecimento acerca da SAE alguns a descreveram como uma ferramenta de organização do CC, confundindo-a com normas e regras do próprio setor de trabalho.

[...]"Eu não lembro exatamente dessa palavra sistematização de enfermagem"[...]"tem todo um sistema de entrada do paciente. Desde a marcação até levar o paciente para cirurgia [...] puncionar veia, todo esse sistema." (TE2)

[...] "todo setor tem seu sistema, suas normas, então procuramos trabalhar sempre dentro do que é passado pra gente." (TE3)

[...]"seriam mais as regras, as normas." (AE2)

[...]"Uma Padronização do serviço? Acho que é isso." (TE4)

"É o cuidado pré- operatório? [...] Ver quais são os sintomas dele, manter ele monitorado. Ver se ele não tem nenhum adorno, e ver se é o apêndice mesmo que ele vai operar." (TE7)

Outros a confundiram com a segurança a ser transmitida ao cliente, o que, na verdade, é um dos resultados esperados com a implementação da SAE.

[...]"Ah é o acolhimento do paciente, passar segurança para ele" (TE1)

[...]" Porque é tão rápido o primeiro cuidado com ele, que a gente tem mesmo o cuidado dele não ficar tão ansioso, de conseguirmos passar um conforto." (TE5)

Durante as entrevistas alguns profissionais evidenciaram o desconhecimento sobre a $S A E$, pois não souberam explicá-la ou, até mesmo, nunca ouviram falar.

"Ah com esse nome eu não conheço não". (TE1)

"Eu desconheço, não ouvi falar nada ainda". (AE1)

"Não lembro o que é sistematização da assistência de Enfermagem "(TE5)

Os enfermeiros, por sua vez, demonstram reconhecer a SAE como importante processo de assistência ao cliente no CC. Percebe-se que já estudaram ou leram sobre a SAE em algum momento de sua trajetória profissional ou acadêmica, porém, demonstraram dificuldade no entendimento de suas fases e aplicação.

[...]"É muito melhor você trabalhar com a SAE. Você primeiro prescreve, a equipe executa e você pode avaliar e evoluir os resultados."(E2)

"A gente já ouviu falar na faculdade sobre a SAE né? Onde existe todo um processo de prescrição de Enfermagem, cuidados de Enfermagem. A SAE ajudaria também no processo para nós enfermeiros." (E1)

[...]"Os cuidados que a gente deve ter perante o paciente né? Tanto na evolução, no histórico do paciente, na avaliação, no exame físico né?" (E3)

\section{O dia a dia da equipe de Enfermagem e a sua influência na aplicação da SAE no CC}

Com relação à influência da rotina dos participantes na aplicação da SAE, percebemos fatores que dificultam sua implementação. Dentre as dificuldades listadas estavam falta de recursos humanos, cirurgias de emergência, falta de comunicação, quantidade de cirurgias, entre outros.

"Falta de comunicação, isso prejudica. Stress de Enfermagem acaba influenciando bastante. A pressa, a correria." (TE1)

"A quantidade de cirurgia, fica corrido, tudo por cima, às vezes mal feito pela quantidade de cirurgia." (TE4)

[...]"Não temos tempo, falta funcionário." (TE9) 
"O problema é que é um setor muito agitado [...] é uma sobrecarga muito grande. Eu tenho problemas com pacientes que são mal preparados lá fora." (AE1)

\section{DISCUSSÃO}

Foi possível perceber que nem todos os participantes demonstraram conhecer a SAE. O dado é preocupante, visto que esta é uma importante ferramenta de trabalho do enfermeiro e sua equipe. Muitos profissionais acreditam que a sistematização é realizada apenas para benefício do cliente e que o processo é utilizado somente para aliviar sua ansiedade e tratá-lo de maneira adequada.

Apesar de haver confusão da SAE com outros processos e rotinas realizadas no $\mathrm{CC}$, a segurança e bem estar do cliente foi bastante citada como parte integrante deste processo, porém não foi relacionada como uma das finalidades no cuidado.

O cliente cirúrgico vivencia o estresse de forma intensa, com medos, ansiedades, preocupações e incertezas, daí a importância da atuação do enfermeiro no sentido de perceber essas manifestações no período pré-operatório ${ }^{(8)}$ e, consequentemente, deixa-lo seguro e confortável.

Para o profissional implementar a SAE em seu ambiente de trabalho é necessário o conhecimento sobre sua utilização. Estudo realizado em Recife objetivou analisar o conhecimento dos enfermeiros sobre a SAE, e verificou que $33 \%$ dos entrevistados apresentavam conhecimento sobre a temática e somente $31,5 \%$ descreveram todas as etapas que a compõe, de forma correta ${ }^{(9)}$.

Em contrapartida, uma pesquisa em Goiânia relatou que todos os participantes da pesquisa conheciam a SAE. Porém foi possível perceber a fragmentação da sua visão, pois citaram como etapas que a compõe somente a prescrição e evolução de enfermagem, o que não é suficiente ${ }^{(10)}$.

Desse modo, o presente estudo, sobretudo, mostra que muitos participantes conhecem, mas não aplicam a SAE, a descrevem de forma incompleta, ou ainda nunca ouviram falar. Nenhum dos participantes deixou claro que ela é o planejamento das ações de Enfermagem, acreditam na importância da sistematização que, segundo os mesmos, melhora a qualidade da assistência, porém salientam que não conseguem implementá-la.

Surge então a dúvida sobre a responsabilidade pela falta de informação e conhecimento e os motivos que impedem a aplicação da SAE. Talvez uma falha na formação ou orientação das equipes coordenadoras/supervisoras dos serviços de saúde; a ausência de estímulo dos gestores; e lacunas na formação dos profissionais, sejam possíveis causas do não conhecimento sobre a $\mathrm{SAE}^{(11-12)}$.

As dificuldades encontradas pelos participantes corroboram com as encontradas na literatura, enfatizando que para realizar este processo o profissional de Enfermagem necessita de base científica, conhecimento, habilidades e atitudes pautadas no compromisso ético e na responsabilidade em assumir o cuidado ${ }^{(12) .}$.

$O$ déficit de recursos materiais e humanos é descrito como a principal barreira para a efetivação da SAE. As dificuldades encontradas induzem os profissionais a acharem que a SAE não é prioridade e ressaltam que deve haver melhoria das condições de trabalho para, posteriormente, implementar essa metodologia ${ }^{(13)}$.

A participação destes profissionais em todas as etapas da SAE é de relevante, sobretudo nos momentos de planejamento de sua implementação no setor, o que os torna mais adeptos à prática da sistematização, e assegura maior usabilidade da ferramenta ${ }^{(14-15)}$.

O enfermeiro é reconhecido pelos demais profissionais da saúde como um profissional integrador de diferentes saberes, principalmente por estar presente junto ao cliente vinte e quatro horas e poder detectar as alterações que acontecem com ele. Neste ponto de vista, a SAE torna-se um canal de comunicação multiprofissional em que o enfermeiro é quem estabelece a ponte de informações entre os membros da equipe garantindo a qualidade da assistência ${ }^{(16)}$.

\section{CONSIDERAÇÕES FINAIS}

Entende-se o CC como um setor complexo e a SAE colabora na organização da prestação do cuidado ao cliente, em momento singular de sua vida. Ressalta-se dificuldades em descrever corretamente a SAE, assim como o entendimento insuficiente sobre o assunto. Porém, alguns compreendem que o processo de Enfermagem poderia prover bons resultados para o dia a dia de trabalho, e que situações não previstas, capazes de impedir ou comprometer o procedimento cirúrgico, poderiam não acontecer se a SAE fosse implantada.

A dificuldade dos enfermeiros em aplicar a SAE em seu local de trabalho é justificada, muitas vezes, pela falta de entendimento sobre o assunto ou por motivos institucionais. Destaca-se a importância de uma padronização do serviço, além da necessidade da educação continuada para que os profissionais atualizem-se, discutam e reflitam formas de aplicação do cuidado de maneira organizada e eficiente 


\section{Referências}

1.Tannure MC; Gonçalves AMP. SAE - Sistematização da Assistência de Enfermagem: Guia Prático. Rio de Janeiro: Guanabara Koogan; 2007.

2. Amante LN, Rosseto AP, Schneider DG. Sistematização da Assistência de Enfermagem em Unidade de Terapia Intensiva sustentada pela Teoria de Wanda Horta. Rev. Esc. Enfermagem; USP. 2009 [Citado 2012 Fev. 10]; 43(1): 54-64. Disponível em: http://www.scielo.br/pdf/reeusp/v43n1/07.pdf

3. Horta W.A. Processo de enfermagem / Wanda de Aguiar Horta, com a colaboração de Brigitta E. P. Castellanos. - São Paulo: EPU; 1979.

4. Truppel TC, Meier MJ, Calixto RC, Peruzzo AS, Crozeta K. Sistematização da Assistência de Enfermagem em Unidade de Terapia Intensiva. Rev. Bras. Enfermagem. 2009, 62(2):221-27 [Citado 2012 Fev. 10]

5. Cofen. Resolução do Conselho Federal de Enfermagem 358/2009, que dispõe sobre Sistematização da Assistência de Enfermagem e a implementação do Processo de Enfermagem. 2009 [Citado 2012 Mar. 13] Disponível em: http://site.portalcofen.gov.br/node/4384.

6. Polit DF, Hungler BP. Fundamentos de pesquisa em enfermagem. $3^{\text {a }}$ ed. Porto Alegre: Artes Médicas, 1995. 7. Bardin L. Análise de conteúdo. Lisboa: Edições 70; 2010. 8. Stumm EMF, Maçalai RT, Kirchner RM. Dificuldades enfrentadas por enfermeiros em um centro cirúrgico. Texto contexto - enfermagem. 2006 [Citado 2012 Out 24]; 15(3): 464-71. Disponível em: http://www.scielo.br/pdf/tce/v15n3/ v15n3a11.pdf.

9. Silva EGC, Oliveira VC, Neves GBC, Guimarães TMR. O conhecimento do enfermeiro sobre a Sistematização da Assistência de Enfermagem: da teoria à prática. Rev. esc. enferm. USP [periódico na Internet]. 2011 [citado 2012 Out 08]; 45(6): 1380-1386. Disponível em: http://www.scielo.br/ pdf/reeusp/v45n6/v45n6a15.pdf
10. Uchôa MG, Lemes MMDD. A visão dos enfermeiros que trabalham em unidade hospitalar pública sobre a Sistematização da Assistência de Enfermagem [Internet]. Goiânia: Núcleo de Estudos e Pesquisas em Saúde e Sociedade (NEPSS); 2008 [citado 2012 abr. 14]. Disponível em: http://www.ucg.br/ucg/institutos/ nepss/monografia/monografia_01.pdf

12. Menezes SRT, Priel MR, Pereira LL. Autonomia e vulnerabilidade do enfermeiro na prática da Sistematização da Assistência de Enfermagem. Rev Esc Enferm USP 2011;45(4):9538. [Citado 2012 Set. 20] Disponível em: http://www.scielo.br/pdf/ reeusp/v45n4/v45n4a23.pdf.

13. Mangueira S.O., De lima J.T.S., Costa S.L.A., Da Nobrega M.M.L., Lopes M.V.O. Implantação da sistematização da assistência de enfermagem: opinião de uma equipe de enfermagem hospitalar. Enfermagem em Foco 2012. (Citado 2012 Out 12); 3(3): 135-38. Disponível em: http://revista.portalcofen.gov.br/index.php/ enfermagem/article/view/298.

14. Malucelli A, Otemaier KR, Bonnet M, Cubas MR, Garcia TR. Sistema de informação para apoio à Sistematização da Assistência de Enfermagem. Rev. Bras. Enferm. 2010 (citado 2012 Out 15); 63(4):629-36. Disponível em: http://www.scielo.br/pdf/reben/ v63n4/20.pdf.

15. Cruz AMP, Almeida M. Competências na formação de Técnicos de Enfermagem para implementar a Sistematização da Assistência de Enfermagem. Rev. Esc. Enfermagem. USP [periódico na Internet]. 2010 [citado 2012 Out 12]; 44(4):921-27. Disponível em: http://www.scielo.br/pdf/reeusp/v44n4/09.pdf

16. Nascimento KC, Backes DS, Koerich MS, Erdmann AL. Sistematização da assistência de enfermagem: vislumbrando um cuidado interativo, complementar e multiprofissional. Rev. esc. enferm. USP [periódico na Internet]. 2008 [citado 2012 Out 12]; 42(4):643-48. Disponível em: http://www.scielo.br/pdf/reeusp/ v42n4/v42n4a04.pdf. 